\title{
Long-Lived and Transient Supersolid Behaviors in Dipolar Quantum Gases
}

\author{
L. Chomaz, ${ }^{1}$ D. Petter, ${ }^{1}$ P. Ilzhöfer, ${ }^{2}$ G. Natale, ${ }^{1}$ A. Trautmann, ${ }^{2}$ C. Politi, ${ }^{2}$ G. Durastante, ${ }^{1,2}$ R. M. W. van Bijnen, ${ }^{2}$ \\ A. Patscheider, ${ }^{1}$ M. Sohmen, ${ }^{1,2}$ M. J. Mark, ${ }^{1,2}$ and F. Ferlaino ${ }^{1,2, *}$ \\ ${ }^{1}$ Institut für Experimentalphysik, Universität Innsbruck, Technikerstraße 25, 6020 Innsbruck, Austria \\ ${ }^{2}$ Institut für Quantenoptik und Quanteninformation, Österreichische Akademie der Wissenschaften, \\ Technikerstraße 21a, 6020 Innsbruck, Austria
}

(Received 11 March 2019; published 19 April 2019; corrected 25 April 2019)

\begin{abstract}
By combining theory and experiments, we demonstrate that dipolar quantum gases of both ${ }^{166} \mathrm{Er}$ and ${ }^{164}$ Dy support a state with supersolid properties, where a spontaneous density modulation and a global phase coherence coexist. This paradoxical state occurs in a well-defined parameter range, separating the phases of a regular Bose-Einstein condensate and of an insulating droplet array, and is rooted in the roton mode softening, on the one side, and in the stabilization driven by quantum fluctuations, on the other side. Here, we identify the parameter regime for each of the three phases. In the experiment, we rely on a detailed analysis of the interference patterns resulting from the free expansion of the gas, quantifying both its density modulation and its global phase coherence. Reaching the phases via a slow interaction tuning, starting from a stable condensate, we observe that ${ }^{166} \mathrm{Er}$ and ${ }^{164} \mathrm{Dy}$ exhibit a striking difference in the lifetime of the supersolid properties, due to the different atom loss rates in the two systems. Indeed, while in ${ }^{166} \mathrm{Er}$ the supersolid behavior survives only a few tens of milliseconds, we observe coherent density modulations for more than $150 \mathrm{~ms}$ in ${ }^{164} \mathrm{Dy}$. Building on this long lifetime, we demonstrate an alternative path to reach the supersolid regime, relying solely on evaporative cooling starting from a thermal gas.
\end{abstract}

DOI: 10.1103/PhysRevX.9.021012

\section{INTRODUCTION}

Supersolidity is a paradoxical quantum phase of matter where both crystalline and superfluid order coexist [1-3]. Such a counterintuitive phase, featuring rather antithetic properties, has been originally considered for quantum crystals with mobile bosonic vacancies, the latter being responsible for the superfluid order. Solid ${ }^{4} \mathrm{He}$ has long been considered a prime system to observe such a phenomenon $[4,5]$. However, after decades of theoretical and experimental efforts, an unambiguous proof of supersolidity in solid ${ }^{4} \mathrm{He}$ is still missing [6,7].

In search of more favorable and controllable systems, ultracold atoms emerged as a very promising candidate, thanks to their highly tunable interactions. Theoretical works point to the existence of a supersolid ground state in different cold-atom settings, including dipolar [8]

\footnotetext{
*Corresponding author.

Francesca.Ferlaino@uibk.ac.at

Published by the American Physical Society under the terms of the Creative Commons Attribution 4.0 International license. Further distribution of this work must maintain attribution to the author(s) and the published article's title, journal citation, and DOI.
}

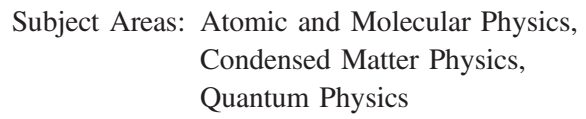

and Rydberg particles $[9,10]$, cold atoms with a softcore potential [11], or lattice-confined systems [7]. Breakthrough experiments with Bose-Einstein condensates (BECs) coupled to light have recently demonstrated a state with supersolid properties $[12,13]$. While in these systems indeed two continuous symmetries are broken, the crystal periodicity is set by the laser wavelength, making the supersolid incompressible.

Another key notion concerns the close relation between a possible transition to a supersolid ground state and the existence of a local energy minimum at large momentum in the excitation spectrum of a nonmodulated superfluid, known as the roton mode [14]. Since excitations corresponding to a periodic density modulation at the roton wavelength are energetically favored, the existence of this mode indicates the system's tendency to crystallize [15] and it is predicted to favor a transition to a supersolid ground state $[4,5,9]$.

Remarkably, BECs of highly magnetic atoms, in which the particles interact through the long-range and anisotropic dipole-dipole interaction (DDI), appear to gather several key ingredients for realizing a supersolid phase. First, as predicted more than 15 years ago $[16,17]$ and recently demonstrated in experiments $[18,19]$, the partial attraction in momentum space due to the DDI gives rise to a roton 
minimum. The corresponding excitation energy, i.e., the roton gap, can be tuned in the experiments down to vanishing values. Here, the excitation spectrum softens at the roton momentum and the system becomes unstable. Second, there is a nontrivial interplay between the trap geometry and the phase diagram of a dipolar BEC. For instance, our recent observations have pointed out the advantage of axially elongated trap geometries (i.e., cigar shaped) compared to the typically considered cylindrically symmetric ones (i.e., pancake shaped) in enhancing the visibility of the roton excitation in experiments. Last but not least, while the concept of a fully softened mode is typically related to instabilities and disruption of a coherent quantum phase, groundbreaking works in the quantum-gas community have demonstrated that quantum fluctuations can play a crucial role in stabilizing a dipolar BEC [20-26]. Such a stabilization mechanism enables the existence, beyond the mean-field instability, of a variety of stable ground states, from a single macrodroplet $[22,24,27]$ to striped phases [28], and droplet crystals [29]; see also related works [30-33]. For multidroplet ground states, efforts have been devoted to understanding if a phase coherence among ground-state droplets could be established $[28,29]$. However, previous experiments with ${ }^{164} \mathrm{Dy}$ have shown the absence of phase coherence across the droplets [28], probably due to the limited atom numbers.

Droplet ground states, quantum stabilization, and dipolar rotons have caused a huge amount of excitement with very recent advancements adding key pieces of information to the supersolid scenario. The quench experiments in an ${ }^{166} \mathrm{Er} \mathrm{BEC}$ at the roton instability have revealed out-ofequilibrium modulated states with an early-time phase coherence over a timescale shorter than a quarter of the oscillation period along the weak-trap axis [18]. In the same work, it has been suggested that the roton softening combined with the quantum stabilization mechanism may open a promising route towards a supersolid ground state. A first confirmation came from a recent theoretical work [34], considering an Er BEC in an infinite elongated trap with periodic boundary conditions and tight transverse confinement. The supersolid phase appears to exist within a narrow region in interaction strength, separating a roton excitation with a vanishing energy and an incoherent assembly of insulating droplets. Almost simultaneously, experiments with ${ }^{162}$ Dy BECs in a shallow elongated trap, performing a slow tuning of the contact interaction, reported on the production of stripe states with phase coherence persisting up to half of the weak trapping period [35]. More recently, such observations have been confirmed in another ${ }^{162}$ Dy experiment [36]. Here, theoretical calculations showed the existence of a phase-coherent droplet ground state, linking the experimental findings to the realization of a state with supersolid properties. The results on ${ }^{162}$ Dy show, however, transient supersolid properties whose lifetime is limited by fast inelastic losses caused by three-body collisions $[35,36]$. These realizations raise the crucial question of whether a long-lived or stationary supersolid state can be created despite the usually non-negligble atom losses and the crossing of a discontinuous phase transition, which inherently creates excitations in the system.

In this work, we study both experimentally and theoretically the phase diagram of degenerate gases of highly magnetic atoms beyond the roton softening. Our investigations are carried out using two different experimental setups producing BECs of ${ }^{166} \mathrm{Er}[22,37]$ and of ${ }^{164} \mathrm{Dy}$ [38] and rely on a fine-tuning of the contact-interaction strength in both systems. In the regime of interest, these two atomic species have different contact-interaction scattering lengths $a_{s}$, whose precise dependence on the magnetic field is known only for $\operatorname{Er}[18,22,39]$, and different three-body-loss rate coefficients. Moreover, Er and Dy possess different magnetic moments $\mu$ and masses $m$, yielding the dipolar lengths, $a_{\mathrm{dd}}=\mu_{0} \mu^{2} m / 12 \pi \hbar^{2}$, of $65.5 a_{0}$ and $131 a_{0}$, respectively. Here, $\mu_{0}$ is the vacuum permeability, $\hbar=h / 2 \pi$ the reduced Planck constant, and $a_{0}$ the Bohr radius. For both systems, we find states showing hallmarks of supersolidity, namely, the coexistence of density modulation and global phase coherence. For such states, we quantify the extent of the $a_{s}$ parameter range for their existence and study their lifetime. For ${ }^{166} \mathrm{Er}$, we find results very similar to the one recently reported for ${ }^{162} \mathrm{Dy}[35,36]$, both systems being limited by strong three-body losses, which destroy the supersolid properties in about half of a trap period. However, for ${ }^{164} \mathrm{Dy}$, we have identified an advantageous magnetic-field region where losses are very low and large BECs can be created. In this condition, we observe that the supersolid properties persist over a remarkably long time, well exceeding the trap period. Based on such a high stability, we finally demonstrate a novel route to reach the supersolid state, based on evaporative cooling from a thermal gas.

\section{THEORETICAL DESCRIPTION}

As a first step in our study of the supersolid phase in dipolar BECs, we compute the ground-state phase diagram for both ${ }^{166} \mathrm{Er}$ and ${ }^{164} \mathrm{Dy}$ quantum gases. The gases are confined in a cigar-shaped harmonic trap, as illustrated in Fig. 1(a). Our theory is based on numerical calculations of the extended Gross-Pitaevskii equation [40], which includes our anisotropic trapping potential, the short-range contact and long-range dipolar interactions at a mean-field level, as well as the first-order beyond-mean-field correction in the form of a Lee-Huang-Yang (LHY) term $[18,22-24,27]$. We note that, while both the exact strength of the LHY term and its dependence on the gas characteristics are under debate $[18,19,25,31,41]$, the importance of such a term, scaling with a higher power in density, is essential for stabilizing states beyond the mean-field instability [18,25,41]; see also Refs. [8,42-44]. 


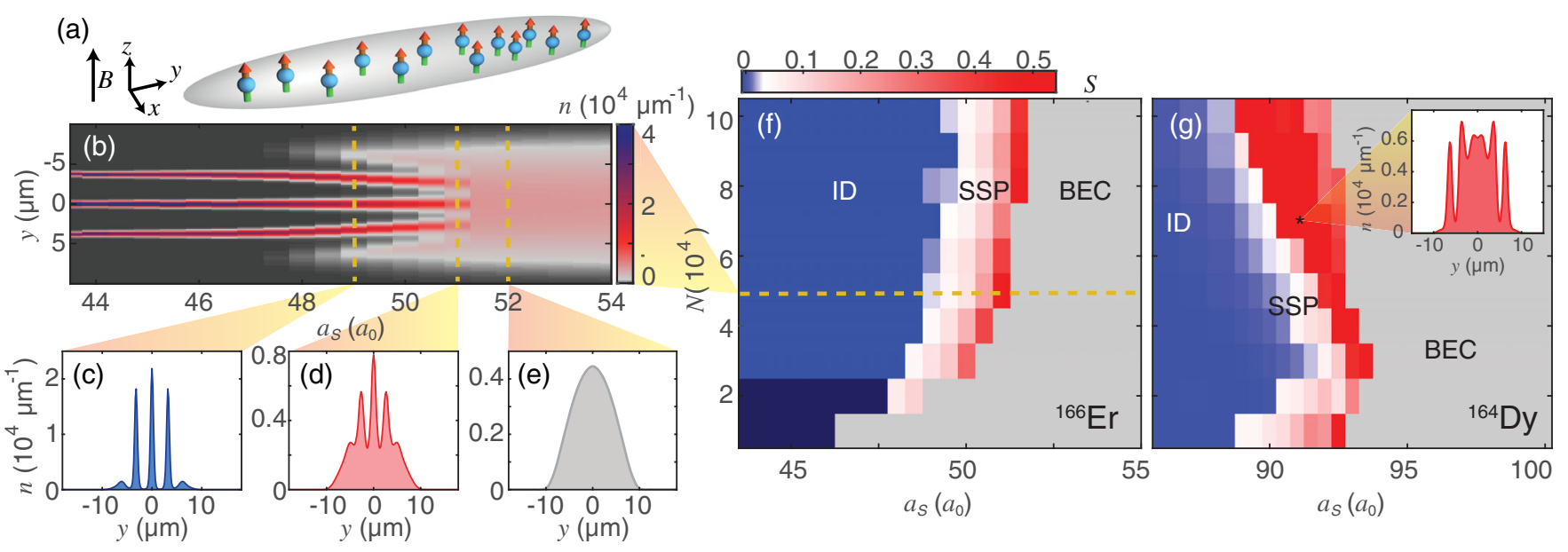

FIG. 1. Phase diagram of an ${ }^{166} \mathrm{Er}$ and a ${ }^{164} \mathrm{Dy}$ dipolar BEC in a cigar-shaped trap. (a) Illustration of the trap geometry with atomic dipoles oriented along $z$. (b) Integrated density profile as a function of $a_{s}$ for an ${ }^{166} \mathrm{Er}$ ground state of $N=5 \times 10^{4}$. In the color bar, the density scale is upper limited to $4 \times 10^{4} \mu \mathrm{m}^{-1}$ in order to enhance the visibility in the supersolid regime. (c)-(e) Exemplary density profiles for an insulating droplet state (ID) at $a_{s}=49 a_{0}$, for a state with supersolid properties (SSP) at $51 a_{0}$, and for a BEC at 52 $a_{0}$, respectively. (f),(g) Phase diagrams for ${ }^{166} \mathrm{Er}$ and ${ }^{164} \mathrm{Dy}$ for trap frequencies $\omega_{x, y, z}=2 \pi \times(227,31.5,151)$ and $2 \pi \times(225,37,135) \mathrm{Hz}$, respectively. The gray color identifies ground states with a single peak in $n(y)$ of large Gaussian width, $\sigma_{y}>2 \ell_{y}$. The dark blue region in (f) shows the region where $n(y)$ exhibits a single sharp peak, $\sigma_{y} \leq 2 \ell_{y}$, and no density modulation. The red-to-blue color map shows $S$ in the case of a density-modulated $n(y)$. In (g) the color map is upper limited to use the same color code as in (f) and to enhance visibility in the low- $N$ regime. The inset in (g) shows the calculated density profile for ${ }^{164} \mathrm{Dy}$ at $N=7 \times 10^{4}$ and $a_{s}=91 a_{0}$.

Our theoretical results are summarized in Fig. 1. By varying the condensed-atom number $N$ and $a_{s}$, the phase diagram shows three very distinct phases. To illustrate them, we first describe the evolution of the integrated in situ density profile $n(y)$ with fixed $N$ for varying $a_{s}$, Fig. 1(b). The first phase, appearing at large $a_{s}$, resembles a regular dilute BEC. It corresponds to a nonmodulated density profile of low peak density and large axial size $\sigma_{y}$ exceeding several times the corresponding harmonic oscillator length $\left(\ell_{y}=\sqrt{\hbar / m \omega_{y}}\right)$; see Fig. 1(e) and the region denoted BEC in Figs. 1(f) and $1(\mathrm{~g})$. The second phase appears when decreasing $a_{s}$ down to a certain critical value, $a_{s}^{*}$. Here, the system undergoes an abrupt transition to a periodic density-modulated ground state, consisting of an array of overlapping narrow droplets, each of high peak density. Because the droplets are coupled to each other via a density overlap, later quantified in terms of the link strength $S$, particles can tunnel from one droplet to a neighboring one, establishing a global phase coherence across the cloud; see Fig. 1(d). Such a phase, in which periodic density modulation and phase coherence coexist, is identified as the supersolid (SSP) one [10,34]; see the SSP region in Figs. 1(f) and 1(g). When further decreasing $a_{s}$, we observe a fast reduction of the density overlap, which eventually vanishes; see Fig. 1(c). Here, the droplets become fully separated. Under realistic experimental conditions, it is expected that the phase relation between such droplets cannot be maintained; see later discussion. We identify this third phase as the one of an insulating droplet (ID) array
$[27,28,45]$; see the ID region in Figs. 1(f) and 1(g). For low $N$, we find a single droplet of high peak density, as in Refs. [24,27]; see dark blue region in Fig. 1(f). Generally speaking, our calculations show that the number of droplets in the array decreases with lowering $a_{s}$ or $N$. The existence of these three phases (BEC, SSP, ID) is consistent with recent calculations considering an infinitely elongated Er BEC [34] and a cigar-shaped ${ }^{162} \mathrm{Dy}$ BEC [36], illustrating the generality of this behavior in dipolar gases.

To study the supersolid character of the density-modulated phases, we compute the average of the wave function overlap between neighboring droplets $S$. As an ansatz to extract $S$, we use a Gaussian function to describe the wave function of each individual droplet. This is found to be an appropriate description from an analysis of the density profiles of Figs. 1(b)-1(d); see also Ref. [46]. For two droplets at a distance $d$ and of identical Gaussian widths $\sigma_{y}$ along the array direction, $S$ is simply $S=\exp \left(-d^{2} / 4 \sigma_{y}^{2}\right)$. Here, we generalize the computation of the wave function overlap to account for the difference in widths and amplitudes among neighboring droplets. This analysis allows us to distinguish between the two types of modulated ground states, SSP and ID in Figs. 1(f) and 1(g). Within the Josephson-junction picture [47-49], the tunneling rate of atoms between neighboring droplets depends on the wave function overlap, and an estimate for the singleparticle tunneling rate can be derived within the Gaussian approximation [46]; see also Ref. [40]. The ID phase corresponds to vanishingly small values of $S$, yielding 
tunneling times extremely long compared to any other relevant timescale. In contrast, the supersolid phase is identified by a substantial value of $S$, with a correspondingly short tunneling time.

As shown in Figs. 1(f) and 1(g), a comparative analysis of the phase diagram for ${ }^{166} \mathrm{Er}$ and ${ }^{164} \mathrm{Dy}$ reveals similarities between the two species (see also Ref. [36]). A supersolid phase is found for sufficiently high $N$, in a narrow region of $a_{s}$, upper bounded by the critical value $a_{s}{ }^{*}(N)$. For intermediate $N, a_{s}^{*}$ increases with increasing $N$. We note that, for low $N$, the nonmodulated BEC evolves directly into a single droplet state for decreasing $a_{s}$ [50]. In this case, no supersolid phase is found in between; see also Refs. [24,27]. Despite the general similarities, we see that the supersolid phase for ${ }^{164} \mathrm{Dy}$ appears for lower atom number than for Er and has a larger extension in $a_{s}$. This is mainly due to the different $a_{\mathrm{dd}}$ and strength of the LHY term. We note that, at large $N$ and for decreasing $a_{s}$, Dy exhibits ground states with a density modulation appearing first in the wings, which then progresses inwards until a substantial modulation over the whole cloud is established [51]; see inset of Fig. 1(g). In this regime, we also observe that $a_{s}^{*}$ decreases with increasing $N$. These types of states have not been previously reported and, although challenging to access in experiments because of the large $N$, they deserve further theoretical investigations.

\section{EXPERIMENTAL SEQUENCE FOR ${ }^{166}$ Er AND ${ }^{164}$ Dy}

To experimentally access the above-discussed physics, we produce dipolar BECs of either ${ }^{166} \mathrm{Er}$ or ${ }^{164} \mathrm{Dy}$ atoms. These two systems are created in different setups and below we summarize the main experimental steps; see also Ref. [40].

Erbium.-We prepare a stable ${ }^{166} \mathrm{Er}$ BEC following the scheme of Ref. [18]. At the end of the preparation, the $\mathrm{Er}$ BEC contains about $N=8 \times 10^{4}$ atoms at $a_{s}=64.5 a_{0}$. The sample is confined in a cigar-shaped optical dipole trap with harmonic frequencies $\omega_{x, y, z}=$ $2 \pi \times(227,31.5,151) \mathrm{Hz}$. A homogeneous magnetic field $B$ polarizes the sample along $z$ and controls the value of $a_{s}$ via a magnetic Feshbach resonance (FR) $[18,22,40]$. Our measurements start by linearly ramping down $a_{s}$ within $20 \mathrm{~ms}$ and waiting an additional $15 \mathrm{~ms}$ so that $a_{s}$ reaches its target value [40]. We note that ramping times between 20 and $60 \mathrm{~ms}$ have been tested in the experiment and we do not record a significant difference in the system's behavior. After the 15-ms stabilization time, we then hold the sample for a variable time $t_{h}$ before switching off the trap. Finally, we let the cloud expand for $30 \mathrm{~ms}$ and perform absorption imaging along the $z$ (vertical) direction, from which we extract the density distribution of the cloud in momentum space, $n\left(k_{x}, k_{y}\right)$.

Dysprosium. - The experimental procedure to create a ${ }^{164}$ Dy BEC follows the one described in Ref. [38]; see also
Ref. [40]. Similarly to Er, the Dy BEC is also confined in a cigar-shaped optical dipole trap and a homogeneous magnetic field $B$ sets the quantization axis along $z$ and the value of $a_{s}$. For Dy, we will discuss our results in terms of magnetic field $B$, since the $a_{s}$-to- $B$ conversion is not well known in the magnetic-field range considered $[25,40,41,52]$. In a first set of measurements, we first produce a stable BEC of about $N=3.5 \times 10^{4}$ condensed atoms at a magnetic field of $B=2.5 \mathrm{G}$ and then probe the phase diagram by tuning $a_{s}$. Here, before ramping the magnetic field to access the interesting $a_{s}$ regions, we slowly increase the power of the trapping beams within $200 \mathrm{~ms}$. The final trap frequencies are $\omega_{x, y, z}=2 \pi \times$ $(300,16,222) \mathrm{Hz}$. After preparing a stable BEC, we ramp $B$ to the desired value within $20 \mathrm{~ms}$ and hold the sample for $t_{h}$ [40]. In a second set of measurements, we study a completely different approach to reach the supersolid state. As discussed later, here we first prepare a thermal sample at a $B$ value where supersolid properties are observed and then further cool the sample until a transition to a coherent droplet-array state is reached. In both cases, at the end of the experimental sequence, we perform absorption imaging after typically $27 \mathrm{~ms}$ of time-of-flight (TOF) expansion. The imaging beam propagates horizontally under an angle $\alpha$ of $\approx 45^{\circ}$ with respect to the weak axis of the trap $(y)$. From the TOF images, we thus extract $n\left(k_{Y}, k_{z}\right)$ with $k_{Y}=\cos (\alpha) k_{y}+\sin (\alpha) k_{x}$.

A special property of ${ }^{164} \mathrm{Dy}$ is that its background scattering length is smaller than $a_{\mathrm{dd}}$. This allows us to enter the supersolid regime without the need of setting $B$ close to a FR, as is done for ${ }^{166} \mathrm{Er}$ and ${ }^{162} \mathrm{Dy}$, which typically causes severe atom losses due to increased threebody-loss coefficients. In contrast, in the case of ${ }^{164} \mathrm{Dy}$, the supersolid regime is reached by ramping $B$ away from the FR pole used to produce the stable BEC via evaporative cooling, as the $a_{s}$ range of Fig. 1(g) lies close to the background $a_{s}$ reported in Ref. [52]; see also Ref. [40]. At the background level, three-body-loss coefficients below $1.3 \times 10^{-41} \mathrm{~m}^{6} \mathrm{~s}^{-1}$ have been reported for ${ }^{164} \mathrm{Dy}$ [25].

\section{DENSITY MODULATION AND PHASE COHERENCE}

The coexistence of density modulation and phase coherence is the key feature that characterizes the supersolid phase and allows us to discriminate it from the BEC and ID cases. To experimentally probe this aspect in our dipolar quantum gases, we record their density distribution after a TOF expansion for various values of $a_{s}$ across the phase diagram. As for a BEC in a weak optical lattice [53] or for an array of BECs [54-56], the appearance of interference patterns in the TOF images is associated with a density modulation of the in situ atomic distribution. Moreover, the shot-to-shot reproducibility of the patterns (in amplitude and position) and the persistence of fringes in averaged 

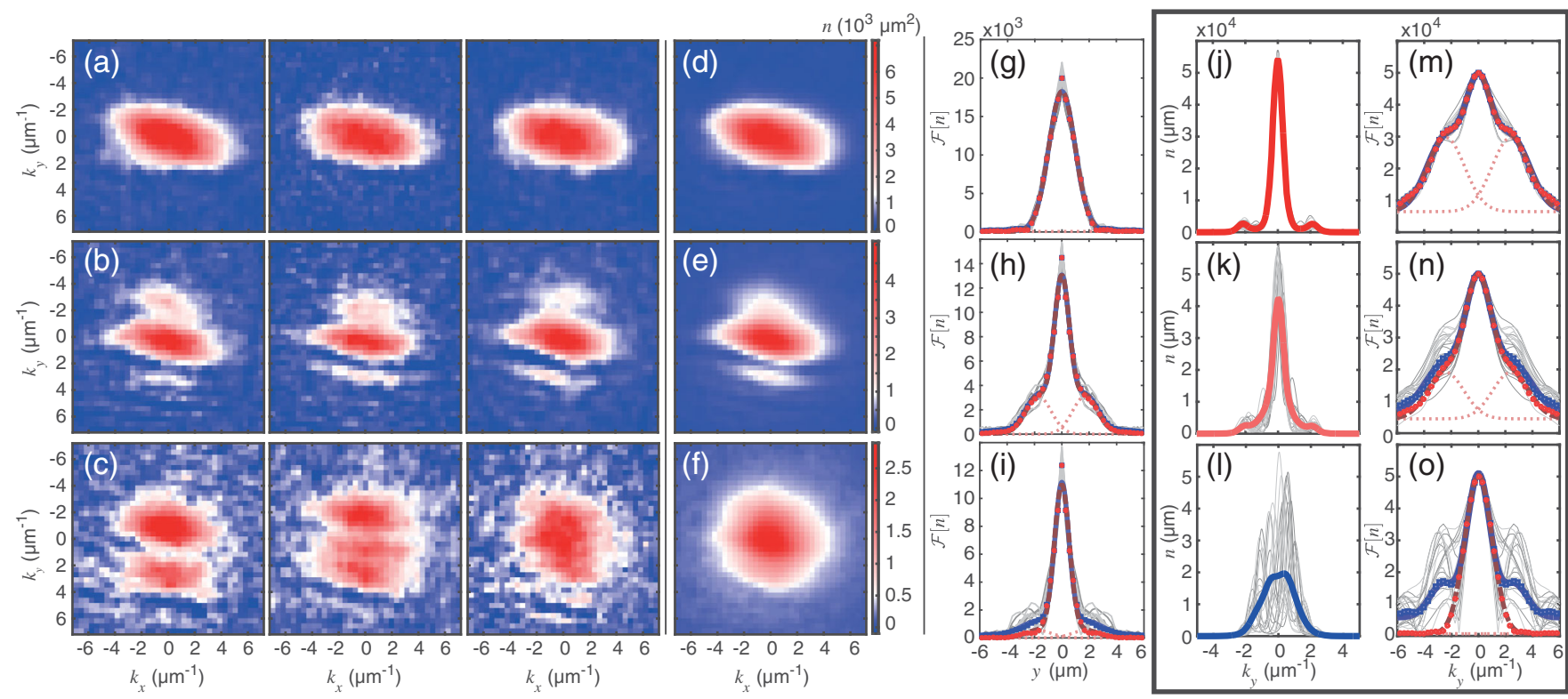

FIG. 2. Coherence in the interference patterns: measurement and toy model. (a)-(c) Examples of single TOF absorption images at $t_{h}=5 \mathrm{~ms}$ for ${ }^{166} \mathrm{Er}$ at $a_{s}=\{54.7(2), 53.8(2), 53.3(2)\} a_{0}$, respectively. Corresponding average pictures for 100 images obtained under the same experimental conditions (d)-(f) and their Fourier transform (FT) profiles (g)-(i). The gray lines show the FT norm $|\mathcal{F}[n](y)|$ of the individual profiles. The averages, $n_{\mathcal{M}}$ (blue squares) and $n_{\Phi}$ (red dots), are fitted to three-Gaussian functions (blue solid line and brown dashed line, respectively). The dotted lines show the components of the total fitted function corresponding to the two side peaks in $n_{\Phi}$. (j)-(1) Interference patterns from the toy-model realizations with 100 independent draws using $N_{D}=4, d=2.8 \mu \mathrm{m}, \sigma_{y}=$ $0.56 \mu \mathrm{m}$ (see text) and for different $\phi_{i}$ distributions: (j) $\phi_{i}=0,(\mathrm{k}) \phi_{i}$ normally distributed around 0 with $0.2 \pi$ standard deviation, (1) $\phi_{i}$ uniformly distributed between 0 and $2 \pi$. (m)-(o) Corresponding FT profiles for the toy model, same color code as (g)-(i).

pictures, obtained from many repeated images taken under the same experimental conditions, reveals the presence of phase coherence across the sample [56].

Figure 2 exemplifies snapshots of the TOF distributions for $\mathrm{Er}$, measured at three different $a_{s}$ values; see Figs. 2(a)-2(c). Even if very close in scattering length, the recorded $n\left(k_{x}, k_{y}\right)$ shows a dramatic change in behavior. For $a_{s}=54.7(2) a_{0}$, we observe a nonmodulated distribution with a density profile characteristic of a dilute BEC. When lowering $a_{s}$ to 53.8(2) $a_{0}$, we observe the appearance of an interference pattern in the density distribution, consisting of a high central peak and two almost symmetric low-density side peaks [57]. Remarkably, the observed pattern is very reproducible with a high shot-to-shot stability, as shown in the repeated single snapshots and in the average image [Figs. 2(b) and 2(e)]. This behavior indicates a coexistence of density modulation and global phase coherence in the in situ state, as expected in the supersolid phase. This observation is consistent with our previous quench experiments [18] and with the recent ${ }^{162}$ Dy experiments $[35,36]$. When further lowering $a_{s}$ to 53.3(2) $a_{0}$, complicated patterns develop with fringes varying from shot to shot in number, position, and amplitude, signaling the persistence of in situ density modulation. However, the interference pattern is completely washed out in the averaged density profiles [Fig. 2(f)], pointing to the absence of a global phase coherence. We identify this behavior as the one of ID states.

Toy model-To get an intuitive understanding of the interplay between density modulation and phase coherence and to estimate the role of the different sources of fluctuations in our experiment, we here develop a simple toy model, which is inspired by Ref. [56]; see also Ref. [40]. In our model, the initial state is an array of $N_{D}$ droplets containing in total $N$ atoms. Each droplet is described by a one-dimensional Gaussian wave function $\psi_{i}(y)$ of amplitude $\alpha_{i}$, phase $\phi_{i}$, width $\sigma_{i}$, and center $y_{i}$. To account for fluctuations in the experiments, we allow $\alpha_{i}$, $d_{i}=y_{i}-y_{i-1}$, and $\sigma_{i}$ to vary by $10 \%$ around their expectation values. The spread of the phases $\phi_{i}$ among the droplets is treated specially as it controls the global phase coherence of the array. By fixing $\phi_{i}=0$ for each droplet or by setting a random distribution of $\phi_{i}$, we range from full phase coherence to the incoherent cases. Therefore, the degree of phase incoherence can be varied by changing the standard deviation of the distribution of $\phi_{i}$.

To mimic our experiment, we compute the free evolution of each individual $\psi_{i}$ over $30 \mathrm{~ms}$, and then compute the axial distribution $n(y, t)=\left|\sum_{i} \psi_{i}(y, t)\right|^{2}$, from which we extract the momentum distribution $n\left(k_{y}\right)$, also accounting for the finite imaging resolution [40]. For each computation run, we randomly draw $N_{D}$ values for $\phi_{i}$, as well as of $\sigma_{i}$, $d_{i}$, and $\alpha_{i}$, and extract $n\left(k_{y}\right)$. We then collect a set of $n\left(k_{y}\right)$ 
by drawing these values multiple times using the same statistical parameters and compute the expectation value, $\left\langle n\left(k_{y}\right)\right\rangle$; see Figs. 2(j)-2(1). The angled brackets denote the ensemble average.

The results of our toy model show large similarity with the observed behavior in the experiment. In particular, while for each single realization one can clearly distinguish multipeak structures regardless of the degree of phase coherence between the droplets, the visibility of the interference pattern in the averaged $n\left(k_{y}\right)$ survives only if the standard deviation of the phase fluctuations between droplets is small (roughly, below $0.3 \pi$ ). In the incoherent case, we note that the shape of the patterns strongly varies from shot to shot. Interestingly, the toy model also shows that the visibility of the coherent peaks in the average images is robust against the typical shot-to-shot fluctuations in droplet size, amplitude, and distance that occur in the experiments; see Figs. 2(j) and 2(k).

Probing density modulation and phase coherence.-To separate and quantify the information on the in situ density modulation and its phase coherence, we analyze the measured interference patterns in Fourier space [36,58-60]. Here, we extract two distinct averaged density profiles, $n_{\mathcal{M}}$ and $n_{\Phi}$. Their structures at finite $y$ spatial frequency (i.e., in Fourier space) quantify the two abovementioned properties.

More precisely, we perform a Fourier transform (FT) of the integrated momentum distributions $n\left(k_{y}\right)$ denoted $\mathcal{F}[n](y)$. Generally speaking, modulations in $n\left(k_{y}\right)$ induce peaks at finite spatial frequency, $y=y^{*}$, in the FT norm, $|\mathcal{F}[n](y)|$; see Figs. 2(g)-2(i) and 2(m)-2(o). Following the above discussion (see also Refs. [56,61]), such peaks in an individual realization hence reveal a density modulation of the corresponding in situ state, with a wavelength roughly equal to $y^{*}$. Consequently, we consider the average of the FT norm of the individual images, $n_{\mathcal{M}}(y)=\langle|\mathcal{F}[n](y)|\rangle$, as the first profile of interest. The peaks of $n_{\mathcal{M}}$ at finite $y$ then indicate the mere existence of an in situ density modulation of roughly constant spacing within the different realizations. As the second profile of interest, we use the FT norm of the average profile $\left\langle n\left(k_{y}\right)\right\rangle, n_{\Phi}(y)=|\mathcal{F}[\langle n\rangle](y)|$. Connecting to our previous discussion, the peaks of $n_{\Phi}$ at finite $y$ point to the persistence of a modulation in the average $\left\langle n\left(k_{y}\right)\right\rangle$, which we identified as a hallmark for a global phase coherence within the density-modulated state. In particular, we point out that a perfect phase coherence, implying identical interference patterns in all the individual realizations, yields $n_{\mathcal{M}}=n_{\Phi}$ and, thus, identical peaks at finite $y$ in both profiles. We note that, by linearity, $n_{\Phi}$ also matches the norm of the average of the full FT of the individual images, i.e., $n_{\Phi}(y)=|\langle\mathcal{F}[n](y)\rangle|$; see also Ref. [40].

Figures 2(g)-2(i) and 2(m)-2(o) demonstrate the significance of our FT analysis scheme by applying it to the momentum distributions from the experiment [Figs. 2(d)-2(f)] and the momentum distributions from the toy model [Figs. 2(j)-2(1)], respectively. As expected, for the BEC case, both $n_{\mathcal{M}}$ and $n_{\Phi}$ show a single peak at zero spatial frequency, $y=0$, characterizing the absence of density modulation, Fig. 2(g). In the case of phase-coherent droplets, Fig. 2(e), we observe that $n_{\mathcal{M}}$ and $n_{\Phi}$ are superimposed and both show two symmetric side peaks at finite $y$, in addition to a dominant peak at $y=0$; see Fig. 2(h). In the incoherent droplet case, we find that, while $n_{\mathcal{M}}$ still shows side peaks at finite $y$, the ones in $n_{\Phi}$ wash out from the averaging, Figs. 2(f), 2(i), 2(l), and 2(o). For both coherent and incoherent droplet arrays, the toy-model results show behaviors matching the above description, providing a further justification of our FT analysis scheme; see Figs. 2(j)-2(o). Our toy model additionally proves two interesting features. First, it shows that the equality $n_{\mathcal{M}}=n_{\Phi}$, revealing the global phase coherence of a density-modulated state, is remarkably robust to noise in the structure of the droplet arrays; see Figs. 2(j) and 2(m). Second, our toy model, however, shows that phase fluctuations across the droplet array on the order of $0.2 \pi$ standard deviation are already sufficient to make $n_{\Phi}$ and $n_{\mathcal{M}}$ deviate from each other; see Figs. 2(k) and 2(n). The incoherent behavior is also associated with strong variations in the side peak amplitude of the individual realizations of $|\mathcal{F}[n]|$, connecting, e.g., to the observations of Ref. [36].

Finally, to quantify the density modulation and the phase coherence, we fit a three-Gaussian function to both $n_{\mathcal{M}}(y)$ and $n_{\Phi}(y)$ and extract the amplitudes of the finite-spatial-frequency peaks, $A_{\mathcal{M}}$ and $A_{\Phi}$, for both distributions, respectively. Note that for a BEC, which is a phase-coherent state, $A_{\Phi}$ will be zero since it probes only finite-spatial-frequency peaks; see Figs. 2(g)-2(i) and $2(\mathrm{~m})-2(\mathrm{o})$.

\section{CHARACTERIZATION OF THE SUPERSOLID STATE}

We are now in the position to study two key aspects, namely, (i) the evolution of the density modulation and phase coherence across the BEC-supersolid-ID phases and (ii) the lifetime of the coherent density-modulated state in the supersolid regime.

Evolution of the supersolid properties across the phase diagram.-The first type of investigation is conducted with ${ }^{166} \mathrm{Er}$ since, contrary to ${ }^{164} \mathrm{Dy}$, its scattering length and dependence on the magnetic field has been precisely characterized [18,22]. After preparing the sample, we ramp $a_{s}$ to the desired value and study the density patterns as well as their phase coherence by probing the amplitudes $A_{\mathcal{M}}$ and $A_{\Phi}$ as a function of $a_{s}$ after $t_{h}=5 \mathrm{~ms}$. As shown in Fig. 3(a), in the BEC region (i.e., for large $a_{s}$ ), we observe that both $A_{\mathcal{M}}$ and $A_{\Phi}$ are almost zero, evidencing the expected absence of a density modulation in the system. As soon as $a_{s}$ reaches a critical value $a_{s}^{*}$, the system's behavior dramatically changes with a sharp and simultaneous 

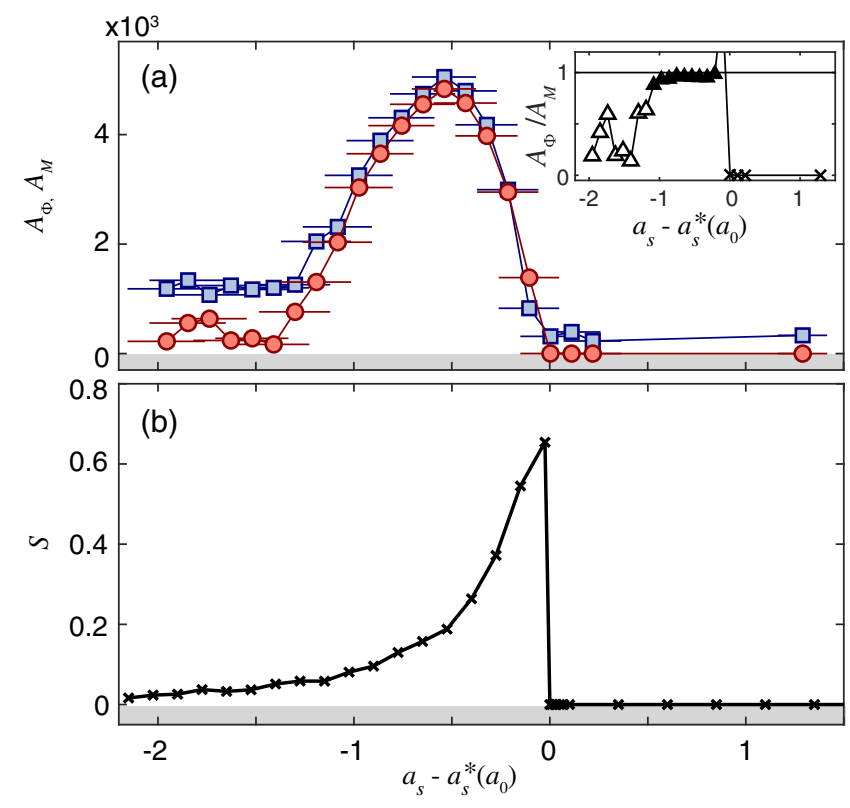

FIG. 3. Supersolid behavior across the phase diagram. Measured side peak amplitudes, $A_{\Phi}$ (circles) and $A_{\mathcal{M}}$ (squares), with their ratio in inset (a), and calculated link strength $S$ (b) as a function of $a_{s}-a_{s}^{*}$ for ${ }^{166} \mathrm{Er}$. For nonmodulated states, we set $S=0$ in theory and $A_{\Phi} / A_{\mathcal{M}}=0$ in experiment (crosses in inset). In the inset, open and closed symbols correspond to $A_{\Phi} / A_{\mathcal{M}}>$ 0.8 and $\leq 0.8$, respectively. In the experiments, we probe the system at a fixed $t_{h}=5 \mathrm{~ms}$. Horizontal error bars are derived from our experimental uncertainty in $B$, vertical error bars corresponding to the statistical uncertainty from the fit are smaller than the data points. The measured and calculated critical scattering lengths are $a_{s}^{*}=54.9(2) a_{0}$ and $51.15 a_{0}$, respectively [62]. The numerical results are obtained for the experimental trap frequencies and for a constant $N=5 \times 10^{4}$ [63].

increase of both $A_{\mathcal{M}}$ and $A_{\Phi}$. While the strength of $A_{\mathcal{M}}$ and $A_{\Phi}$ varies with decreasing $a_{s}$-first increasing then decreasing - we observe that their ratio $A_{\Phi} / A_{\mathcal{M}}$ remains constant and close to unity over a narrow $a_{s}$ range below $a_{s}^{*}$ of $\gtrsim 1 a_{0}$ width; see the inset of Fig. 3(a). This behavior pinpoints the coexistence in the system of phase coherence and density modulation, as predicted to occur in the supersolid regime. For $\left(a_{s}-a_{s}^{*}\right)<-1 a_{0}$, we observe that the two amplitudes depart from each other. Here, while the density modulation still survives with $A_{\mathcal{M}}$ saturating to a lower finite value, the global phase coherence is lost with $A_{\Phi} / A_{\mathcal{M}}<1$, as expected in the insulating droplet phase. Note that we also study the evolution of $A_{\Phi}$ and $A_{\mathcal{M}}$ in ${ }^{164} \mathrm{Dy}$, but as a function of $B$, and find a qualitatively similar behavior.

To get a deeper insight on how our observations compare to the phase-diagram predictions (see Fig. 1), we study the link strength $S$ as a function of $a_{s}$; see Fig. 3(b). Since $S$ quantifies the density overlap between neighboring droplets and is related to the tunneling rate of atoms across the droplet array, it thus provides information on the ability of the system to establish or maintain a global phase coherence. In this plot, we set $S=0$ in the case where no modulation is found in the ground state. At the BEC-tosupersolid transition, i.e., at $a_{s}=a_{s}^{*}$, a density modulation abruptly appears in the system's ground state with $S$ taking a finite value. Here, $S$ is maximal, corresponding to a density modulation of minimal amplitude. Below the transition, we observe a progressive decrease of $S$ with lowering $a_{s}$, pointing to the gradual reduction of the tunneling rate in the droplet arrays. Close to the transition, we estimate a large tunneling compared to all other relevant timescales. However, we expect this rate to become vanishingly small, on the sub-Hertz level [40], when decreasing $a_{s} 1-2 a_{0}$ below $a_{s}^{*}$. Our observation also hints at the smooth character of the transition from a supersolid to an ID phase.

The general trend of $S$, including the extension in $a_{s}$ where it takes nonvanishing values, is similar to the $a_{s}$ behavior of $A_{\mathcal{M}}$ and $A_{\Phi}$ observed in the experiments [62]. We observe in the experiments that the $a_{s}$ dependence at the BEC-to-supersolid transition appears sharper than at the supersolid-to-ID interface, potentially suggesting a different nature of the two transitions. However, more investigations are needed since atom losses, finite temperature, and finite-size effects can affect, and in particular smoothen, the observed behavior [64-66]. Moreover, dynamical effects, induced by, e.g., excitations created at the crossing of the phase transitions or atom losses during the time evolution, can also play a substantial role in the experimental observations, complicating a direct comparison with the ground-state calculations. The time dynamics as well as a different scheme to achieve a state with supersolid properties is the focus of the remainder of the paper.

Lifetime of the supersolid properties.-Having identified the $a_{s}$ range in which our dipolar quantum gas exhibits supersolid properties, the next central question concerns the stability and lifetime of such a fascinating state. Recent experiments on ${ }^{162}$ Dy have shown the transient character of the supersolid properties, whose lifetime is limited by three-body losses [35,36]. In these experiments, the phase coherence is found to survive up to $20 \mathrm{~ms}$ after the density modulation has formed. This time corresponds to about half of the weak-trap period. Stability is a key issue in the supersolid regime, especially since the tuning of $a_{s}$, used to enter this regime, has a twofold consequence on the inelastic loss rate. First, it gives rise to an increase in the peak density [see Figs. 1(b)-1(d)] and, second, it may lead to an enhancement of the three-body-loss coefficient.

We address this question by conducting comparative studies on ${ }^{166} \mathrm{Er}$ and ${ }^{164} \mathrm{Dy}$ gases. These two species allow us to tackle two substantially different scattering scenarios. Indeed, the background value of $a_{s}$ for ${ }^{166} \mathrm{Er}$ (as well as for ${ }^{162} \mathrm{Dy}$ ) is larger than $a_{\mathrm{dd}}$. Thus, reaching the supersolid regime, which occurs at $a_{\mathrm{dd}} / a_{s} \approx 1.2-1.4$ in our geometry, requires us to tune $B$ close to the pole of a FR. This tuning 
also causes an increase of the three-body-loss rate. In contrast, ${ }^{164}$ Dy realizes the opposite case with the background scattering length smaller than $a_{\mathrm{dd}}$. This feature brings the important advantage of requiring tuning $B$ away from the FR pole to reach the supersolid regime. As we describe below, this important difference in scattering properties leads to a strikingly longer lifetime of the ${ }^{164} \mathrm{Dy}$ supersolid properties with respect to ${ }^{166} \mathrm{Er}$ and to the recently observed behavior in ${ }^{162}$ Dy $[35,36]$.

The measurements proceed as follows. For both ${ }^{166} \mathrm{Er}$ and ${ }^{164} \mathrm{Dy}$, we first prepare the quantum gas in the stable $\mathrm{BEC}$ regime and then ramp $a_{s}$ to a fixed value in the supersolid regime for which the system exhibits a state of coherent droplets (i.e., $A_{\Phi} / A_{\mathcal{M}} \approx 1$ ); see previous discussion. Finally, we record the TOF images after a variable $t_{h}$ and we extract the time evolution of both $A_{\Phi}$ and $A_{\mathcal{M}}$. The study of these two amplitudes will allow us to answer the question of whether the droplet structure-i.e., the density modulation in space-persists in time whereas the coherence among droplets is lost $\left(A_{\mathcal{M}}>A_{\Phi} \rightarrow 0\right)$ or if the density structures themselves vanish in time $\left(A_{\mathcal{M}} \approx A_{\Phi} \rightarrow 0\right)$.

As shown in Fig. 4, for both species, we observe that $A_{\Phi}$ and $A_{\mathcal{M}}$ decay almost synchronously with a remarkably longer lifetime for ${ }^{164} \mathrm{Dy}$ [Fig. 4(b)] than ${ }^{166} \mathrm{Er}$ [Fig. 4(a)].
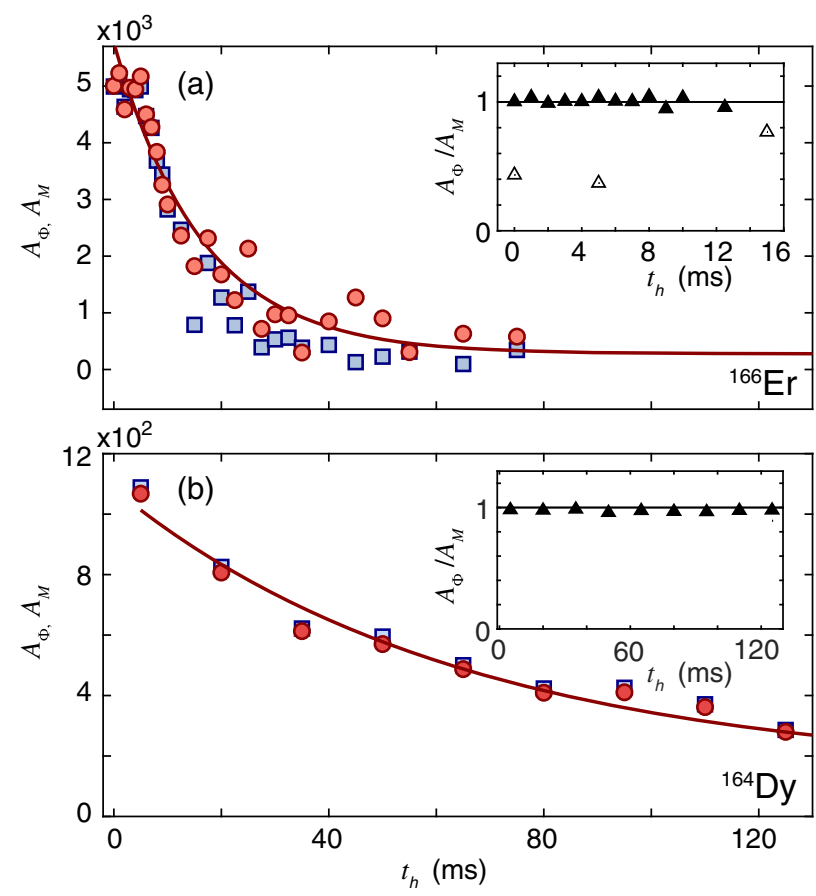

FIG. 4. Time evolution of the supersolid properties. Amplitudes $A_{\Phi}$ (circles) and $A_{\mathcal{M}}$ (squares) in the supersolid regime as a function of the holding time in trap for (a) ${ }^{166} \mathrm{Er}$ at $54.2(2) a_{0}$ and for (b) ${ }^{164} \mathrm{Dy}$ at $2.04 \mathrm{G}$. The solid lines are exponential fits to the data. The insets show the time evolution of $A_{\Phi} / A_{\mathcal{M}}$ for the above cases (filled triangles), and, for comparison, in the ID regime (empty triangles) for Er at $a_{s}=53.1(2) a_{0}$ (a).
Interestingly, $A_{\Phi}$ and $A_{\mathcal{M}}$ remain approximately equal during the whole time dynamics; see insets of Figs. 4(a) and 4(b). This behavior indicates that it is the strength of the density modulation itself and not the phase coherence among droplets that decays over time. Similar results have been found theoretically in Ref. [67]. We connect this decay mainly to three-body losses, especially detrimental for ${ }^{166} \mathrm{Er}$, and possible excitations created while crossing the BEC-to-supersolid phase transition [40]. For comparison, the inset of Fig. 4(a) shows also the behavior in the ID regime for ${ }^{166} \mathrm{Er}$, where $A_{\Phi} / A_{\mathcal{M}}<1$ already at short $t_{h}$ and remains so during the time evolution [40].

To get a quantitative estimate of the survival time of the phase-coherent and density-modulated state, we fit a simple exponential function to $A_{\Phi}$ and extract $t_{\Phi}$, defined as the $1 / 10$ lifetime; see Fig. 4 . For ${ }^{166} \mathrm{Er}$, we extract $t_{\Phi}=38(6) \mathrm{ms}$. For $t_{h}>t_{\Phi}$, the interference patterns become undetectable in our experiment and we recover a signal similar to the one of a nonmodulated BEC state [as in Figs. 2(a) and 2(d)]. These results are consistent with recent observations of transient supersolid properties in ${ }^{162} \mathrm{Dy}$ [35]. For ${ }^{164} \mathrm{Dy}$, we observe that the coherent densitymodulated state is remarkably long-lived. Here, we find $t_{\Phi}=152(13) \mathrm{ms}$.

The striking difference in the lifetime and robustness of the supersolid properties between ${ }^{166} \mathrm{Er}$ and ${ }^{164} \mathrm{Dy}$ becomes even more visible when studying $t_{\Phi}$ as a function of $a_{s}$ ( $B$ for Dy). As shown in Fig. $5, t_{\Phi}$ for Er remains comparatively low in the investigated supersolid regime and slightly varies between 20 and $40 \mathrm{~ms}$. Similarly to the recent studies with ${ }^{162} \mathrm{Dy}$, this finding reveals the transient character of the state and opens the question of whether a stationary supersolid state can be reached with these species. On the contrary, for ${ }^{164}$ Dy we observe that $t_{\Phi}$ first increases with $B$ in the range from $1.8 \mathrm{G}$ to about $1.98 \mathrm{G}$. Then, for $B>1.98 \mathrm{G}, t_{\Phi}$ acquires a remarkably large and almost constant value of about $150 \mathrm{~ms}$ over a wide $B$ range. This shows the long-lived character of the supersolid properties in our ${ }^{164}$ Dy quantum gas. We note that over the investigated range, $a_{s}$ is expected to monotonously increase with $B$ [40]. Such a large value of $t_{\Phi}$ exceeds not only the estimated tunneling time across neighboring droplets but also the weak-axis trap period, which together set the typical timescale to achieve global equilibrium and to study collective excitations.

\section{CREATION OF STATES WITH SUPERSOLID PROPERTIES BY EVAPORATIVE COOLING}

The long-lived supersolid properties in ${ }^{164}$ Dy motivate us to explore an alternative route to cross the supersolid phase transition, namely, by evaporative cooling instead of interaction tuning. For this set of experiments, we have modified the waists of our trapping beams in order to achieve quantum degeneracy in tighter traps with respect to 


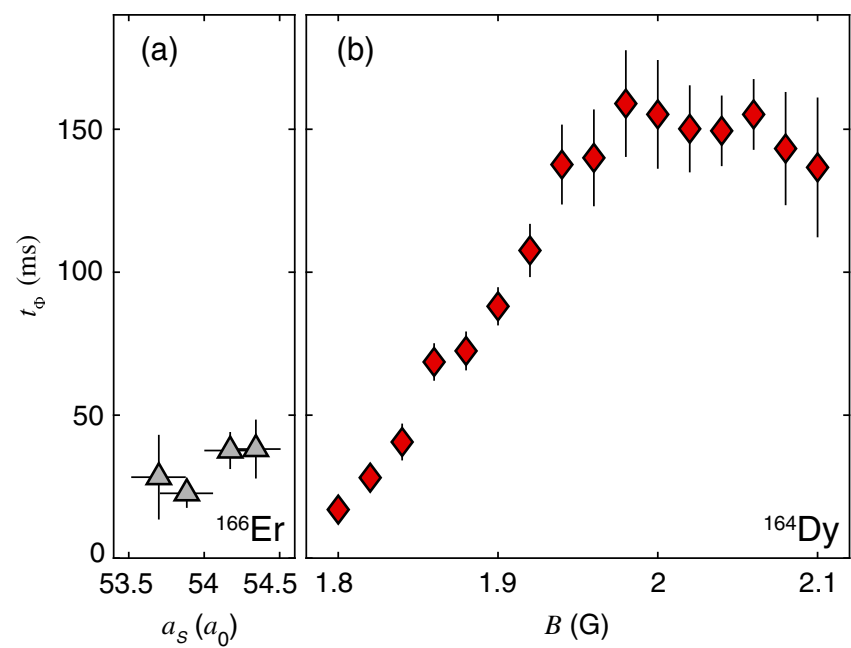

FIG. 5. Survival time of the coherent density-modulated state. $t_{\Phi}$ in ${ }^{166} \mathrm{Er}$ as a function of $a_{s}$ (a) and ${ }^{164} \mathrm{Dy}$ as a function of $B(\mathrm{~b})$. The error bars refer to the statistical uncertainty from the fit. The range of investigation corresponds to the supersolid regime for which phase-coherent density-modulated states are observed. This range is particularly narrow for ${ }^{166} \mathrm{Er}$.

the one used for condensation in the previous set of measurements. In this way, the interference peaks in the supersolid region are already visible without the need to apply a further compression of the trap since the sideto-central-peak distance in the momentum distribution scales roughly as $1 / \ell_{z}$ [18]. Forced evaporative cooling is performed by reducing the power of the trapping beams piecewise linearly in subsequent evaporation steps until a final trap with frequencies $2 \pi \times(225,37,134) \mathrm{Hz}$ is achieved. During the whole evaporation process, which has an overall duration of about $3 \mathrm{~s}$, the magnetic field is kept either at $B=2.43 \mathrm{G}$, where we observe long-lived interference patterns, or at $B=2.55 \mathrm{G}$, where we produce a stable nonmodulated BEC. We note that these two $B$ values are very close without any FR lying in between [40].

Figure 6 shows the phase transition from a thermal cloud to a final state with supersolid properties by evaporative cooling. In particular, we study the phase transition by varying the duration of the last evaporation ramp, while maintaining the initial and final trap-beam power fixed. This procedure effectively changes the atom number and temperature in the final trap while keeping the trap parameters unchanged, which is important to not alter the final ground-state phase diagram of the system. At the end of the evaporation, we let the system equilibrate and thermalize for $t_{h}=100 \mathrm{~ms}$, after which we switch off the trap, let the atoms expand for $26.5 \mathrm{~ms}$, and finally perform absorption imaging. We record the TOF images for different ramp durations, i.e., for different thermalization times. For a short ramp, too many atoms are lost such that the critical atom number for condensation is not reached, and the atomic distribution remains thermal; see Fig. 6(a).

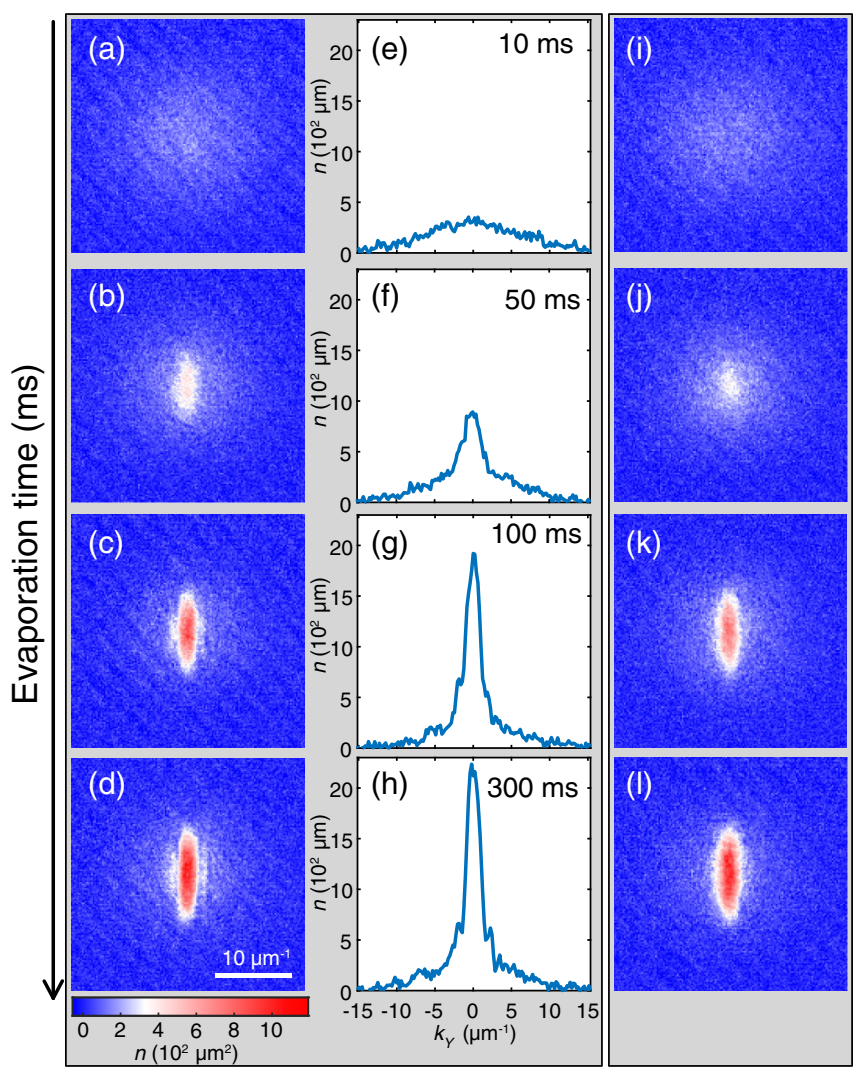

FIG. 6. Evaporative cooling to a state with supersolid properties. ${ }^{164}$ Dy absorption images showing the transition to a state with supersolid properties at $2.43 \mathrm{G}(\mathrm{a})-(\mathrm{d})$ and to a BEC state at $2.55 \mathrm{G}$ (i)-(l), via different durations of the last evaporation step. These durations are $10 \mathrm{~ms}$ (a),(i), $50 \mathrm{~ms}$ (b),(j), $100 \mathrm{~ms}$ (c),(k), and $300 \mathrm{~ms}(\mathrm{~d}),(\mathrm{l})$. The density profiles (e)-(h) are integrated over the central regions of the corresponding absorption images (a)-(d). The color map indicates the atomic density in momentum space.

By increasing the ramp time, the evaporative cooling becomes more efficient and we observe the appearance of a bimodal density profile with a narrow and dense peak at the center, which we identify as a regular BEC; see Fig. 6(b). By further cooling, the BEC fraction increases and the characteristic pattern of the supersolid state emerges; see Figs. 6(c) and 6(d). The observed evaporation process shows a strikingly different behavior in comparison with the corresponding situation at $B=2.55 \mathrm{G}$, where the usual thermal-to-BEC phase transition is observed; see Figs. 6(i)-6(1).

We finally probe the lifetime of the supersolid properties by extracting the time evolution of both the amplitudes $A_{\Phi}$ and $A_{\mathcal{M}}$, as previously discussed. We use the same experimental sequence as the one in Fig. 6(d)—i.e., 300$\mathrm{ms}$ duration of the last evaporation ramp and $100 \mathrm{~ms}$ of equilibration time-and subsequently hold the sample in the trap for a variable $t_{h}$. As shown in Fig. 7(a), we observe a very long lifetime with both amplitudes staying large and almost constant over more than $200 \mathrm{~ms}$. At longer holding 


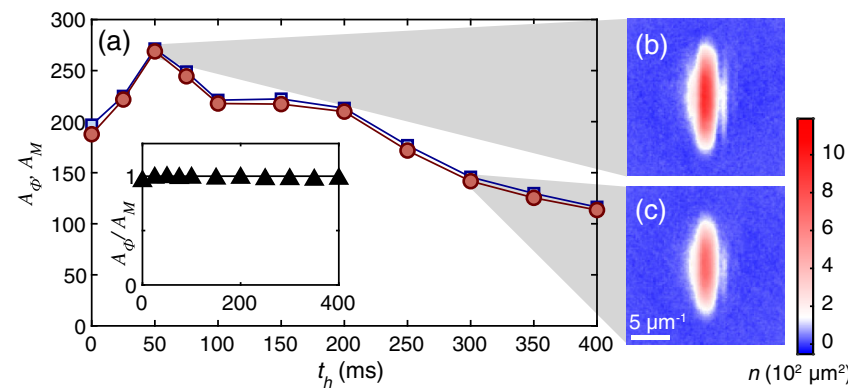

FIG. 7. Lifetime of the supersolid properties achieved via evaporative cooling. Time evolution of the amplitudes $A_{\Phi}$ (red circle) and $A_{\mathcal{M}}$ (square) after an evaporation time of $300 \mathrm{~ms}$ at $2.43 \mathrm{G}$ and an equilibration time of $100 \mathrm{~ms}$. The inset shows the time evolution of $A_{\Phi} / A_{\mathcal{M}}$. At $t_{h}=0 \mathrm{~ms}$, the atom number in the phase-coherent density-modulated component is $N=$ $2.2(2) \times 10^{4}$. (b),(c) Averaged absorption images of 25 realizations after 50 and $300 \mathrm{~ms}$ of holding time, respectively. Note that the thermal background has been subtracted from the images. The color map indicates the atomic density in momentum space.

time, we observe a slow decay of $A_{\Phi}$ and $A_{\mathcal{M}}$, following the decay of the atom number. Moreover, during the dynamics, the ratio $A_{\Phi} / A_{\mathcal{M}}$ stays constant. The long lifetime of the phase-coherent density modulation is also directly visible in the persistence of the interference patterns in the averaged momentum density profiles [similar to Fig. 2(e)], both at intermediate and long times; see Figs. 7(b) and 7(c), respectively. For even longer $t_{h}$, we cannot resolve anymore interference patterns in the TOF images. Here, we recover a signal consistent with a regular BEC of low $N$.

Achieving the coherent droplet phase via evaporative cooling is a very powerful alternative path to supersolidity. We speculate that, for instance, excitations, which might be important when crossing the phase transitions by interaction tuning, may be small or removed by evaporation when reaching this state kinematically. Other interesting questions, open to future investigations, are the nature of the phase transition, the critical atom number, and the role of noncondensed atoms.

\section{CONCLUSIONS}

For both ${ }^{166} \mathrm{Er}$ and ${ }^{164} \mathrm{Dy}$ dipolar quantum gases, we have identified and studied states showing hallmarks of supersolidity, namely, global phase coherence and spontaneous density modulations. These states exist in a narrow scattering-length region, lying between a regular BEC phase and a phase of an insulating droplet array. While for ${ }^{166} \mathrm{Er}$, similarly to the recently reported ${ }^{162}$ Dy case $[35,36]$, the observed supersolid properties fade out over a comparatively short time because of atom losses, we find that ${ }^{164} \mathrm{Dy}$ exhibits remarkably long-lived supersolid properties. Moreover, we are able to directly create stationary states with supersolid properties by evaporative cooling, demonstrating a powerful alternative approach to interaction tuning on a BEC. This novel technique provides prospects of creating states with supersolid properties while avoiding additional excitations and dynamics. The ability to produce long-lived supersolid states paves the way for future investigations on quantum fluctuations and many-body correlations, as well as of collective excitations in such an intriguing many-body quantum state. A central goal of these future investigations lies in proving the superfluid character of this phase, beyond its global phase coherence [7,34,68-70].

\section{ACKNOWLEDGMENTS}

We thank R. Bisset, B. Blakie, M. Boninsegni, G. Modugno, T. Pfau, and, in particular, L. Santos for many stimulating discussions. Part of the computational results presented have been achieved using the HPC infrastructure LEO of the University of Innsbruck. We acknowledge support by the Austrian Science Fund FWF through the DFG/FWF Forschergruppe (FOR 2247/PI2790), by the ERC Consolidator Grant (RARE, No. 681432), and by a NFRI Grant (MIRARE, No. ÖAW0600) from the Austrian Academy of Science. G. D. and M. S. acknowledge support by the Austrian Science Fund FWF within the DK-ALM (No. W1259-N27).

Note added.-Recently, we became aware of related works reporting theoretical studies of the ground-state phase diagram $[71,72]$.

[1] A. F. Andreev and I. M. Lifshitz, Many-Body Problem in Quantum Mechanics and Quantum Statistical Mechanics, Sov. Phys. JETP 29, 1107 (1969).

[2] G. V. Chester, Speculations on Bose-Einstein Condensation and Quantum Crystals, Phys. Rev. A 2, 256 (1970).

[3] A. J. Leggett, Can a Solid Be "Superfluid"?, Phys. Rev. Lett. 25, 1543 (1970).

[4] D. A. Kirzhnits and Y. A. Nepomnyashchii, Coherent Crystallization of Quantum Liquid, Sov. Phys. JETP 32, 1191 (1971).

[5] T. Schneider and C. P. Enz, Theory of the Superfluid-Solid Transition of ${ }^{4} \mathrm{He}$, Phys. Rev. Lett. 27, 1186 (1971).

[6] S. Balibar, The Enigma of Supersolidity, Nature (London) 464, 176 (2010).

[7] M. Boninsegni and N. V. Prokof'ev, Colloquium: Supersolids: What and Where Are They?, Rev. Mod. Phys. 84, 759 (2012).

[8] Z.-K. Lu, Y. Li, D. S. Petrov, and G. V. Shlyapnikov, Stable Dilute Supersolid of Two-Dimensional Dipolar Bosons, Phys. Rev. Lett. 115, 075303 (2015).

[9] N. Henkel, R. Nath, and T. Pohl, Three-Dimensional Roton Excitations and Supersolid Formation in Rydberg-Excited Bose-Einstein Condensates, Phys. Rev. Lett. 104, 195302 (2010). 
[10] F. Cinti, P. Jain, M. Boninsegni, A. Micheli, P. Zoller, and G. Pupillo, Supersolid Droplet Crystal in a Dipole-Blockaded Gas, Phys. Rev. Lett. 105, 135301 (2010).

[11] M. Boninsegni, Supersolid Phases of Cold Atom Assemblies, J. Low Temp. Phys. 168, 137 (2012).

[12] J. Léonard, A. Morales, P. Zupancic, T. Esslinger, and T. Donner, Supersolid Formation in a Quantum Gas Breaking a Continuous Translational Symmetry, Nature (London) 543, 87 (2017).

[13] J.-R. Li, J. Lee, W. Huang, S. Burchesky, B. Shteynas, F. Ç. Top, A. O. Jamison, and W. Ketterle, A Stripe Phase with Supersolid Properties in Spin-Orbit-Coupled BoseEinstein Condensates, Nature (London) 543, 91 (2017).

[14] L. D. Landau, The Theory of Superfluidity of Helium II, J. Phys. (Moscow) 5, 71 (1941).

[15] P. Nozières, Is the Roton in Superfluid ${ }^{4} \mathrm{He}$ the Ghost of a Bragg Spot?, J. Low Temp. Phys. 137, 45 (2004).

[16] D. H. J. O'Dell, S. Giovanazzi, and G. Kurizki, Rotons in Gaseous Bose-Einstein Condensates Irradiated by a Laser, Phys. Rev. Lett. 90, 110402 (2003).

[17] L. Santos, G. V. Shlyapnikov, and M. Lewenstein, RotonMaxon Spectrum and Stability of Trapped Dipolar BoseEinstein Condensates, Phys. Rev. Lett. 90, 250403 (2003).

[18] L. Chomaz, R. M. W. van Bijnen, D. Petter, G. Faraoni, S. Baier, J. H. Becher, M. J. Mark, F. Wächtler, L. Santos, and F. Ferlaino, Observation of Roton Mode Population in a Dipolar Quantum Gas, Nat. Phys. 14, 442 (2018).

[19] D. Petter, G. Natale, R. M. W. van Bijnen, A. Patscheider, M. J. Mark, L. Chomaz, and F. Ferlaino, Probing the Roton Excitation Spectrum of a Stable Dipolar Bose Gas, arXiv: 1811.12115 .

[20] H. Kadau, M. Schmitt, M. Wenzel, C. Wink, T. Maier, I. Ferrier-Barbut, and T. Pfau, Observing the Rosensweig Instability of a Quantum Ferrofluid, Nature (London) 530, 194 (2016).

[21] I. Ferrier-Barbut, H. Kadau, M. Schmitt, M. Wenzel, and T. Pfau, Observation of Quantum Droplets in a Strongly Dipolar Bose Gas, Phys. Rev. Lett. 116, 215301 (2016).

[22] L. Chomaz, S. Baier, D. Petter, M. J. Mark, F. Wächtler, L. Santos, and F. Ferlaino, Quantum-Fluctuation-Driven Crossover from a Dilute Bose-Einstein Condensate to a Macrodroplet in a Dipolar Quantum Fluid, Phys. Rev. X 6 , 041039 (2016).

[23] F. Wächtler and L. Santos, Quantum Filaments in Dipolar Bose-Einstein Condensates, Phys. Rev. A 93, 061603(R) (2016).

[24] F. Wächtler and L. Santos, Ground-State Properties and Elementary Excitations of Quantum Droplets in Dipolar Bose-Einstein Condensates, Phys. Rev. A 94, 043618 (2016).

[25] M. Schmitt, M. Wenzel, F. Böttcher, I. Ferrier-Barbut, and T. Pfau, Self-Bound Droplets of a Dilute Magnetic Quantum Liquid, Nature (London) 539, 259 (2016).

[26] I. Ferrier-Barbut, M. Wenzel, M. Schmitt, F. Böttcher, and T. Pfau, Onset of a Modulational Instability in Trapped Dipolar Bose-Einstein Condensates, Phys. Rev. A 97, 011604(R) (2018).

[27] R. N. Bisset, R. M. Wilson, D. Baillie, and P. B. Blakie, Ground-State Phase Diagram of a Dipolar Condensate with Quantum Fluctuations, Phys. Rev. A 94, 033619 (2016).
[28] M. Wenzel, F. Böttcher, T. Langen, I. Ferrier-Barbut, and T. Pfau, Striped States in a Many-Body System of Tilted Dipoles, Phys. Rev. A 96, 053630 (2017).

[29] D. Baillie and P. B. Blakie, Droplet Crystal Ground States of a Dipolar Bose Gas, Phys. Rev. Lett. 121, 195301 (2018).

[30] D. S. Petrov, Quantum Mechanical Stabilization of a Collapsing Bose-Bose Mixture, Phys. Rev. Lett. 115, 155302 (2015).

[31] C. R. Cabrera, L. Tanzi, J. Sanz, B. Naylor, P. Thomas, P. Cheiney, and L. Tarruell, Quantum Liquid Droplets in a Mixture of Bose-Einstein Condensates, Science 359, 301 (2018).

[32] G. Semeghini, G. Ferioli, L. Masi, C. Mazzinghi, L. Wolswijk, F. Minardi, M. Modugno, G. Modugno, M. Inguscio, and M. Fattori, Self-Bound Quantum Droplets of Atomic Mixtures in Free Space, Phys. Rev. Lett. 120, 235301 (2018).

[33] P. Cheiney, C. R. Cabrera, J. Sanz, B. Naylor, L. Tanzi, and L. Tarruell, Bright Soliton to Quantum Droplet Transition in a Mixture of Bose-Einstein Condensates, Phys. Rev. Lett. 120, 135301 (2018).

[34] S. M. Roccuzzo and F. Ancilotto, Supersolid Behaviour of a Dipolar Bose-Einstein Condensate Confined in a Tube, Phys. Rev. A 99, 041601(R) (2019).

[35] L. Tanzi, E. Lucioni, F. Fama, J. Catani, A. Fioretti, C. Gabbanini, R. N. Bisset, L. Santos, and G. Modugno, Observation of a Dipolar Quantum Gas with Metastable Supersolid Properties, Phys. Rev. Lett. 122, 130405 (2019).

[36] F. Böttcher, J.-N. Schmidt, M. Wenzel, J. Hertkorn, M. Guo, T. Langen, and T. Pfau, Transient Supersolid Properties in an Array of Dipolar Quantum Droplets, Phys. Rev. X 9, 011051 (2019).

[37] K. Aikawa, A. Frisch, M. Mark, S. Baier, A. Rietzler, R. Grimm, and F. Ferlaino, Bose-Einstein Condensation of Erbium, Phys. Rev. Lett. 108, 210401 (2012).

[38] A. Trautmann, P. Ilzhöfer, G. Durastante, C. Politi, M. Sohmen, M. J. Mark, and F. Ferlaino, Dipolar Quantum Mixtures of Erbium and Dysprosium Atoms, Phys. Rev. Lett. 121, 213601 (2018).

[39] S. Baier, M. J. Mark, D. Petter, K. Aikawa, L. Chomaz, Z. Cai, M. Baranov, P. Zoller, and F. Ferlaino, Extended BoseHubbard Models with Ultracold Magnetic Atoms, Science 352, 201 (2016).

[40] See Supplemental Material at http://link.aps.org/ supplemental/10.1103/PhysRevX.9.021012 for details on the experimental setup, measurement schemes, analysis, and theory calculations.

[41] I. Ferrier-Barbut, M. Wenzel, F. Böttcher, T. Langen, M. Isoard, S. Stringari, and T. Pfau, Scissors Mode of Dipolar Quantum Droplets of Dysprosium Atoms, Phys. Rev. Lett. 120, 160402 (2018).

[42] A. Gammal, T. Frederico, L. Tomio, and P. Chomaz, Atomic Bose-Einstein Condensation with Three-Body Interactions and Collective Excitations, J. Phys. B 33, 4053 (2000).

[43] A. Bulgac, Dilute Quantum Droplets, Phys. Rev. Lett. 89, 050402 (2002).

[44] D. S. Petrov, Quantum Mechanical Stabilization of a Collapsing Bose-Bose Mixture, Phys. Rev. Lett. 115, 155302 (2015). 
[45] F. Wächtler and L. Santos, Ground-State Properties and Elementary Excitations of Quantum Droplets in Dipolar Bose-Einstein Condensates, Phys. Rev. A 94, 043618 (2016).

[46] M. Wenzel, F. Böttcher, J.-N. Schmidt, M. Eisenmann, T. Langen, T. Pfau, and I. Ferrier-Barbut, Anisotropic Superfluid Behavior of a Dipolar Bose-Einstein Condensate, Phys. Rev. Lett. 121, 030401 (2018).

[47] B. D. Josephson, Possible New Effects in Superconductive Tunnelling, Phys. Lett. 1, 251 (1962).

[48] J. Javanainen, Oscillatory Exchange of Atoms between Traps Containing Bose Condensates, Phys. Rev. Lett. 57, 3164 (1986).

[49] S. Raghavan, A. Smerzi, S. Fantoni, and S. R. Shenoy, Coherent Oscillations between Two Weakly Coupled BoseEinstein Condensates: Josephson Effects, $\pi$ Oscillations, and Macroscopic Quantum Self-Trapping, Phys. Rev. A 59, 620 (1999).

[50] A similar behavior is also seen for ${ }^{164}$ Dy for $N<4000$ [out of the scale of Fig. 1(g)].

[51] We note that a similar behavior is also found in our ${ }^{166} \mathrm{Er}$ phase diagram but for larger $N$ than the one reported here.

[52] Y. Tang, A. Sykes, N. Q. Burdick, J. L. Bohn, and B. L. Lev, $s$-Wave Scattering Lengths of the Strongly Dipolar Bosons ${ }^{162}$ Dy and ${ }^{164}$ Dy, Phys. Rev. A 92, 022703 (2015).

[53] M. Greiner, O. Mandel, T. Esslinger, T. W. Hänsch, and I. Bloch, Quantum Phase Transition from a Superfluid to a Mott Insulator in a Gas of Ultracold Atoms, Nature (London) 415, 39 (2002).

[54] M. Greiner, I. Bloch, O. Mandel, T. W. Hänsch, and T. Esslinger, Exploring Phase Coherence in a $2 \mathrm{D}$ Lattice of Bose-Einstein Condensates, Phys. Rev. Lett. 87, 160405 (2001).

[55] B. Paredes, A. Widera, V. Murg, O. Mandel, S. Fölling, I. Cirac, G. V. Shlyapnikov, T. W. Hänsch, and I. Bloch, Tonks-Girardeau Gas of Ultracold Atoms in an Optical Lattice, Nature (London) 429, 277 (2004).

[56] Z. Hadzibabic, S. Stock, B. Battelier, V. Bretin, and J. Dalibard, Interference of an Array of Independent BoseEinstein Condensates, Phys. Rev. Lett. 93, 180403 (2004).

[57] In our absorption imaging a residual asymmetry can be observed between $\pm k_{y}$. This asymmetry, providing a sharper peak at $k_{y}>0$, stems from optical aberrations in our imperfect optical setup. These imperfections make the quantitative analysis of our data more challenging, yet they do not affect the quantitative interpretation. In particular, we have checked that the peak structures are also observable along a distinct imaging axis, nonorthogonal to $y$, using an independent imaging setup.

[58] M. Takeda, H. Ina, and S. Kobayashi, Fourier-Transform Method of Fringe-Pattern Analysis for Computer-Based Topography and Interferometry, J. Opt. Soc. Am. 72, 156 (1982).
[59] C. Kohstall, S. Riedl, E. R. S. Guajardo, L. A. Sidorenkov, J. H. Denschlag, and R. Grimm, Observation of Interference between Two Molecular Bose-Einstein Condensates, New J. Phys. 13, 065027 (2011).

[60] L. Chomaz, L. Corman, T. Bienaimé, R. Desbuquois, C. Weitenberg, S. Nascimbène, J. Beugnon, and J. Dalibard, Emergence of Coherence via Transverse Condensation in a Uniform Quasi-Two-Dimensional Bose Gas, Nat. Commun. 6, 6162 (2015).

[61] S. Hofferberth, I. Lesanovsky, B. Fischer, T. Schumm, and J. Schmiedmayer, Non-Equilibrium Coherence Dynamics in One-Dimensional Bose Gases, Nature (London) 449, 324 (2007).

[62] The values of $a_{s}^{*}$ extracted from experiments and from theory disagree by $3.8 a_{0}$. Such a mismatch is similar to the one observed in our previous work on the roton instability $[18,19]$. This behavior might be related to the possibility that beyond-mean-field effects are not properly accounted for by the conventional LHY correction term; see main text. In addition, other effects, such as the dynamics and the atom losses [40], can affect the experimental observations.

[63] This atom number corresponds to the condensed atom number measured within the stabilization time of $a_{s}$ [40].

[64] M. E. Fisher and A. E. Ferdinand, Interfacial, Boundary, and Size Effects at Critical Points, Phys. Rev. Lett. 19, 169 (1967).

[65] Y. Imry and D. Bergman, Critical Points and Scaling Laws for Finite Systems, Phys. Rev. A 3, 1416 (1971).

[66] Y. Imry, Finite-Size Rounding of a First-Order Phase Transition, Phys. Rev. B 21, 2042 (1980).

[67] See the theoretical analysis in the revised version of Ref. [35].

[68] V. W. Scarola, E. Demler, and S. D. Sarma, Searching for a Supersolid in Cold-Atom Optical Lattices, Phys. Rev. A 73, 051601(R) (2006).

[69] Z.-K. Lu, Y. Li, D. S. Petrov, and G. V. Shlyapnikov, Stable Dilute Supersolid of Two-Dimensional Dipolar Bosons, Phys. Rev. Lett. 115, 075303 (2015).

[70] F. Cinti and M. Boninsegni, Classical and Quantum Filaments in the Ground State of Trapped Dipolar Bose Gases, Phys. Rev. A 96, 013627 (2017).

[71] Y. Kora and M. Boninsegni, Patterned Supersolids in Dipolar Bose Systems, arXiv:1902.08256.

[72] Y.-C. Zhang, F. Maucher, and T. Pohl, Supersolidity around a Critical Point in Dipolar Bose Einstein Condensates, arXiv:1903.06161.

Correction: The inadvertent omission of a marker indicating "Featured in Physics" has been fixed. 